\title{
Postgastrectomy gastric cancer patients are at high risk for colorectal neoplasia: a case control study
}

\author{
Tae-Geun Gweon ${ }^{1 *}$, Kyu-Tae Yoon ${ }^{1 *}$, Chang Hyun Kim², Jin-Jo Kim² \\ ${ }^{1}$ Division of Gastroenterology, Department of Internal Medicine and ${ }^{2}$ Department of Surgery, College of Medicine, The Catholic University of \\ Korea, Seoul, Korea
}

Background/Aims: Several studies have shown that colorectal neoplasms (CRN) including colorectal cancer (CRC) may be prevalent in patients with gastric cancer. However, in most of these studies, colonoscopy to investigate the prevalence of CRN was performed prior to surgery. We aimed to investigate whether CRN was more prevalent in postgastrectomy gastric cancer patients than in healthy individuals. Methods: We reviewed the medical records of those patients within a cohort of gastric cancer patients with gastrectomy who underwent colonoscopy between 2016 and 2017. Controls age- and sex-matched with gastric cancer patients at a 2:1 ratio were identified among those who underwent colonoscopy at a health-promotion center. The frequencies of CRN, advanced CRN (ACRN), and CRC among patients with gastrectomy were compared with those in the control subjects. A total of 744 individuals (gastric cancer, 248; control, 496) were included. Results: The rates of CRN and ACRN in the gastric cancer group were higher than those in the healthy individuals (CRN, $47.6 \%$ vs. $34.7 \%, P<0.001$; ACRN, $16.9 \%$ vs. $10.9 \%, P=0.020)$. The rate of CRC was comparable between the 2 groups $(2.0 \%$ vs. $0.6 \%, P=0.125)$. Multivariate analysis identified previous gastrectomy for gastric cancer and male sex as significant risk factors for (A)CRN. Conclusions: CRN and ACRN were more prevalent in patients who underwent surgery for gastric cancer than in the control group. Regular surveillance colonoscopy at appropriate intervals is indicated after gastrectomy. (Intest Res 2021;19:239-246)

Key Words: Stomach neoplasms; Colonoscopy; Colorectal neoplasm; Colonoscopic surveillance

\section{INTRODUCTION}

The prognosis of patients with gastric cancer has improved with detection at early stages and advances in treatment modalities. ${ }^{1,2}$ Therefore, detection of secondary cancer is as important as surveillance of gastric cancer recurrence. Colorectal cancer (CRC) has been recognized as the most common synchronous malignancy in patients with gastric cancer. ${ }^{3-5}$ The prevalence of concomitant CRC at the time of surgical or en-

Received March 9, 2020. Revised May 21, 2020. Accepted June 3, 2020 Correspondence to Chang Hyun Kim, Department of Surgery, Incheon St. Mary's Hospital, College of Medicine, The Catholic University of Korea, 56 Dongsu-ro, Bupyeong-gu, Incheon 21431, Korea. Tel: +82-32-280-5052, Fax: +82-32-280-5987, E-mail: gospel4@catholic.ac.kr

*These authors contributed equally to this study.

This study was presented as an oral poster at IMKASID on April 13-14, 2018, in Seoul, Korea. doscopic treatment in gastric cancer patients was reported as $1 \%-4 \%{ }^{3-6}$ Although the prevalence of colorectal neoplasms (CRN) including CRC was higher in patients with gastric cancer than in healthy individuals, most studies have investigated the prevalence of concomitant CRN at the time of surgical resection or endoscopic resection of gastric cancer. CRC develops from benign CRN through the adenoma-carcinoma sequence. ${ }^{7}$ Regular surveillance colonoscopy based on the number and histology of resected CRN is recommended after resection of $\mathrm{CRN}^{8,9}$ No previous study has investigated the prevalence of CRN after surgical treatment of gastric cancer. In this study, we aimed to investigate whether CRN was more prevalent in gastric cancer patients after surgical treatment than in healthy individuals. 


\section{METHODS}

Among a cohort of gastric adenocarcinoma patients who underwent gastrectomy at Incheon St. Mary's Hospital (Incheon, Republic of Korea), we reviewed the medical records of consecutive subjects who underwent colonoscopy between January 2016 and December 2017. ${ }^{10}$ We excluded patients with cecal intubation failure; or inadequate bowel preparation. Colonoscopy was performed at least 1 year after gastrectomy. In our center, colonoscopy for gastric cancer patients is included in the routine staging work-up before surgery. ${ }^{11}$ Therefore, most gastric cancer patients undergo colonoscopy before surgery. The control group comprised healthy subjects who underwent colonoscopy at the health-care center of our institution during the study period and were matched with gastric cancer patients by age and sex in a 2:1 ratio. Demographic characteristics, bowel preparation quality, and results of colonoscopy were investigated. Cancer staging and type of surgery were investigated in the gastric cancer group. Cancer staging was performed at the time of gastrectomy using the 7th edition of the American Joint Committee on Cancer Staging Manual. ${ }^{12}$

\section{Colonoscopic Examinations}

Colonoscopy was performed by 3 attending physicians with more than 4 years of experience using an Olympus (Olympus, Seoul, Korea) series Q260 or Q290 colonoscope. ${ }^{13,14}$ Bowel preparation was done with $4 \mathrm{~L}$ of polyethylene glycol. To obtain optimal bowel cleansing, patients were instructed to ingest additional liquid until no solid particles were observed in the bowel effluent. ${ }^{15}$ Bowel cleansing quality was assessed using the Aronchick scale. ${ }^{16,17}$ Polyp resection was performed during withdrawal of the colonoscope. The colonoscopists were encouraged to observe the colonic mucosa for at least 6 minutes. ${ }^{18}$ Polyp resection was performed by cold snare polypectomy or conventional endoscopic mucosal resection at the physician's discretion. Histologic assessment of resected polyps was performed by specialist gastrointestinal pathologists. Cecal intubation time, colonoscope withdrawal time, and total colonoscopy time were analyzed. Colonoscope withdrawal time was determined as the time difference between photographic documentation of the cecum and anus in patients who did not undergo any procedures such as a biopsy or polypectomy. ${ }^{19}$

\section{Definition}

CRN included adenomas or cancers but excluded nonneo- plastic lesions (hyperplastic, lymphoid, inflammatory). Advanced CRN (ACRN) was defined when one of the following criteria was met: (1) adenocarcinoma; (2) adenoma sized at least $1 \mathrm{~cm}$; (3) the presence of at least 3 adenomas; and (4) the presence of villous adenoma or high-grade dysplasia. ${ }^{8,20}$ The rate of CRN, ACRN, and CRC and the number of adenomas per patient were analyzed. The protocol of this study was approved by the Institutional Review Board of Incheon St. Mary's Hospital (IRB No. OC17MES0031). Written informed consent was obtained from all patients.

\section{Statistical Analysis}

All statistical analyses were performed using IBM SPSS version 20 (IBM Corp., Armonk, NY, USA). Continuous variables were compared using Student $t$-test or the Mann-Whitney test when appropriate. Categorical variables are presented as number (percent) and compared using the chi-square or Fisher exact tests. We compared the clinical data and prevalence of (A)CRN and CRC between the gastric cancer and the control group. To investigate the factors contributing to having (A) CRN or CRC, we compared the clinical data of all the patients with and without (A)CRN or CRC. Logistic regression modeling was used to perform multivariate analysis of risk factors for (A)CRN or CRC that were found to be significant in the univariate analysis. Odds ratio (OR) and 95\% confidence interval (CI) were calculated for the prevalence of (A)CRN and CRC. A $P$-value $<0.05$ was considered significant.

\section{RESULTS}

Two hundred and sixty-six patients with gastric cancer underwent colonoscopy during the study period. Of these patients, 18 were excluded because of cecal intubation failure $(n=5)$ or inadequate bowel preparation $(n=13)$. After matching of healthy subjects, a total of 744 patients were finally included in this study: gastric cancer $(n=248)$ and healthy control $(n=496)$. The demographic and clinical data of the 2 groups are shown in Table 1. Age, sex, comorbidities, family history of CRC, and factors related to previous colonoscopy were similar between the 2 groups. Body mass index was lower in the patients with gastrectomy (gastric cancer group $21.3 \mathrm{~kg} / \mathrm{m}^{2}$ vs. control group $\left.24.4 \mathrm{~kg} / \mathrm{m}^{2}, P<0.001\right)$. Indications for colonoscopy differed between the 2 groups $(P<0.001)$. The median interval between surgery and colonoscopy in the gastric cancer group was 51 months. 
Table 1. Baseline Characteristics

\begin{tabular}{|c|c|c|c|}
\hline Characteristics & Gastric cancer $(n=248)$ & Control $(n=496)$ & $P$-value \\
\hline Male sex & $148(60.0)$ & $296(60.0)$ & 1.000 \\
\hline \multicolumn{4}{|l|}{ Comorbidity } \\
\hline Diabetes & $47(19.0)$ & $105(20.2)$ & 0.479 \\
\hline Constipation & $15(6.0)$ & $25(5.0)$ & 0.566 \\
\hline Family history of colorectal cancer & $16(6.6)$ & $26(5.2)$ & 0.467 \\
\hline \multicolumn{4}{|l|}{ Factors of previous colonoscopy } \\
\hline Previous history of colonoscopy & $225(90.7)$ & $433(87.3)$ & 0.168 \\
\hline Screening & $117(47.2)$ & $213(42.9)$ & \\
\hline Surveillance & $120(48.4)$ & $202(40.7)$ & \\
\hline Others & $11(4.4)$ & $81(16.3)$ & \\
\hline \multicolumn{4}{|l|}{ Type of gastrectomy } \\
\hline Total gastrectomy & $45(18.1)$ & NA & \\
\hline Subtotal gastrectomy & $203(81.9)$ & NA & \\
\hline Laparoscopic surgery & $158(63.7)$ & NA & \\
\hline \multicolumn{4}{|l|}{ Cancer stage $^{b}$} \\
\hline I & $164(66.1)$ & NA & \\
\hline
\end{tabular}

Values are presented as mean \pm standard deviation, number (\%), or median (interquartile range).

${ }^{a}$ Data were obtained in patients with history of colonoscopy.

${ }^{6}$ Cancer staging was done according to American Joint Committee on Cancer 7th edition. ${ }^{16}$

NA, not applicable.

\section{Prevalence of CRN}

The rate of polyp detection in the gastric cancer group was $55.6 \%$, which was higher than that in the control group (46.4\%, $P=0.017$ ). The prevalence of CRN and ACRN in the gastric cancer group was higher than that in the control group (CRN: $47.6 \%$ vs. $34.7 \%$, OR, $1.710 ; 95 \%$ CI, $1.254-2.332 ; P<0.001$ and ACRN: $16.9 \%$ vs. $10.9 \%$, OR, 1.669; 95\% CI, 1.079-2.580; $P=0.020$ ) (Table 2, Fig. 1). However, the rate of CRC was comparable between the 2 groups (gastric cancer $2 \%$ vs. control $0.6 \%, P=0.125$ ). The rate of sessile serrated adenoma/polyp was comparable between the 2 groups ( $2.4 \%$ vs. $2.0 \%, P=0.721$ ). The mean number of adenomas per patient was comparable between the 2 groups (gastric cancer 0.9 vs. control $0.7, P=0.833$ ). Metastatic cancer was found in 3 gastric cancer patients (Fig. 2). In the gastric cancer group, the rate of CRN (stage I $48.8 \%$ vs. stage II+III $45.2 \%, P=0.597$ ), ACRN (stage I $15.2 \%$ vs. stage II+III $20.2 \%, P=0.321$ ) and CRC (stage I $1.2 \%$ vs. stage II+III $3.6 \%$, $P=0.340$ ) was not higher in the advanced cancer stage. However, all metastatic cancer was found in gastric cancer with stage III.

\section{Results of Colonoscopy}

The details of colonoscopy are shown in Table 2. The time needed for colonoscope insertion was longer in the gastric cancer group than in the control group (gastric cancer 5.0 minutes vs. control 3.9 minutes, $P<0.001$ ). Withdrawal time 
was comparable for both groups (gastric cancer 6.8 minutes vs. control 6.7 minutes, $P=0.386$ ). Total colonoscopy time was longer in the gastric cancer patients (gastric cancer $16.8 \mathrm{~min}$ utes vs. control 14.1 minutes, $P<0.001)$. Overall bowel cleans-

Table 2. Results of Colonoscopy

\begin{tabular}{lccr}
\hline Characteristics & $\begin{array}{c}\text { Gastric } \\
\text { cancer } \\
(\mathrm{n}=248)\end{array}$ & $\begin{array}{c}\text { Control } \\
(\mathrm{n}=496)\end{array}$ & P-value \\
\hline Colorectal neoplasm & & & \\
All colorectal neoplasm & $118(47.6)$ & $172(34.7)$ & $<0.001$ \\
Advanced colorectal neoplasm & $42(16.9)$ & $54(10.9)$ & 0.020 \\
$\quad$ No. of adenoma $\geq 3$ & $30(12.1)$ & $32(6.5)$ & 0.009 \\
$\quad$ Size of adenoma $\geq 1$ cm & $15(6.0)$ & $22(4.4)$ & 0.034 \\
$\quad$ Villous or high-grade dysplasia & $13(5.2)$ & $25(5.0)$ & 0.906 \\
$\quad$ Colorectal cancer & $5(2.0)$ & $3(0.6)$ & 0.125 \\
Sessile serrated adenoma/polyp & $6(2.4)$ & $10(2.0)$ & 0.721 \\
\hline Time related to colonoscopy (min) & & & \\
Insertion time & $5.0 \pm 2.7$ & $3.9 \pm 2.6$ & $<0.001$ \\
Withdrawal time & & & \\
Total procedure time & $6.8 \pm 1.3$ & $6.7 \pm 1.5$ & 0.386 \\
Bowel cleansing & $16.8 \pm 3.4$ & $14.1 \pm 2.7$ & $<0.001$ \\
Excellent & & & $<0.001$ \\
Good & $34(13.7)$ & $166(33.5)$ & \\
Fair & $112(45.2)$ & $217(43.8)$ & \\
\hline
\end{tabular}

Values are presented as number (\%) or mean \pm standard deviation. Withdrawal time was checked in 110 in the gastric cancer group and 266 in the control group. ing efficacy in the control group was superior to that in the patients with gastrectomy $(P<0.001)$. Perforation or postpolypectomy bleeding did not occur in either group.

\section{Risk Factors for CRN}

Male sex and a history of gastrectomy were significant risk factors for CRN in univariate analysis (Table 3). The patients with CRN were older than those without CRN (CRN 63.2 years vs. without CRN 61.9 years, $P=0.042$ ). Multivariate analysis revealed that male sex (OR, 2.183; 95\% CI, 1.590-2.996) and a history of surgery for gastric cancer (OR, 1.748; 95\% CI, 1.274-

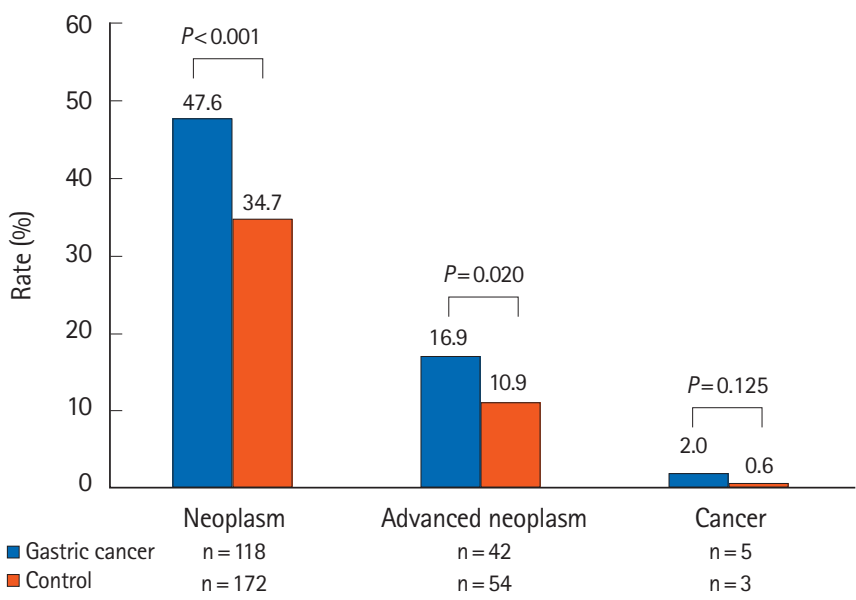

Fig. 1. Comparison of the rate of detection of colorectal neoplasm in the 2 groups. The rates of colorectal neoplasm and advanced colorectal neoplasm were significantly higher in the gastric cancer group than in the control group.
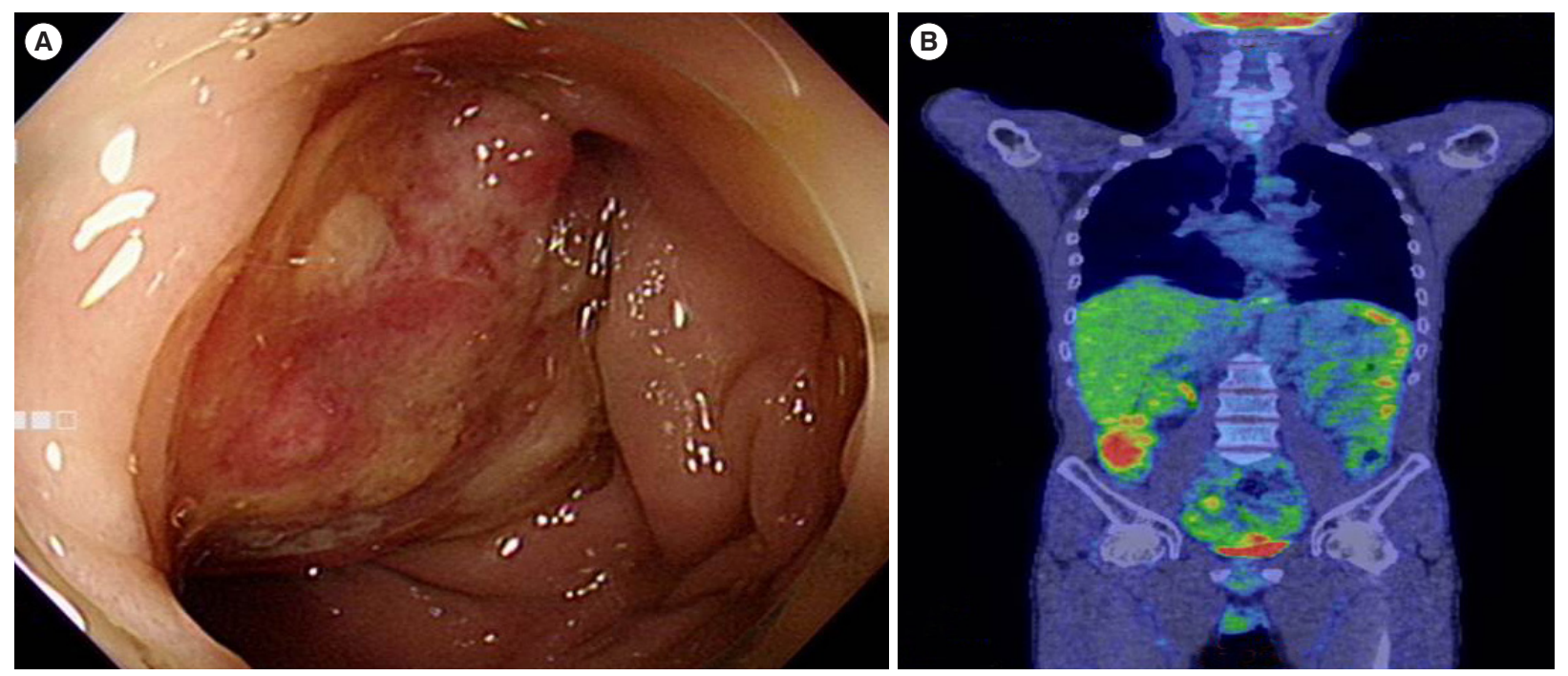

Fig. 2. Detection of metastatic gastric cancer. (A) A luminal obstructing mass was noted during colonoscopy. (B) Positron emission tomography-computed tomography revealed prominent fluorodeoxyglucose uptake at the ascending colon. 
Table 3. Risk Factors of Colorectal Neoplasm of All Study Subjects

\begin{tabular}{|c|c|c|c|c|c|c|}
\hline \multirow{2}{*}{ Characteristics } & \multirow{2}{*}{$\begin{array}{l}\text { Colorectal neoplasm } \\
\qquad(n=290)\end{array}$} & \multirow{2}{*}{$\begin{array}{c}\text { Absent } \\
(n=454)\end{array}$} & \multirow{2}{*}{$P$-value } & \multicolumn{3}{|c|}{ Multivariate analysis } \\
\hline & & & & OR & $95 \% \mathrm{Cl}$ & $P$-value \\
\hline Age (yr) & $63.2 \pm 8.0$ & $61.9 \pm 8.7$ & 0.042 & 0.983 & $0.965-1.001$ & 0.057 \\
\hline Male sex & 205 (70.7) & $239(52.6)$ & $<0.001$ & 2.183 & $1.590-2.996$ & $<0.001$ \\
\hline Body mass index $\left(\mathrm{kg} / \mathrm{m}^{2}\right)$ & $23.5 \pm 3.2$ & $23.4 \pm 3.3$ & 0.621 & & & \\
\hline Diabetes & $60(20.7)$ & $92(20.3)$ & 0.888 & & & \\
\hline Family history of colorectal cancer & $20(6.9)$ & $22(4.8)$ & 0.237 & & & \\
\hline History of colorectal polyp & $100(34.5)$ & $186(41.0)$ & 0.076 & & & \\
\hline Gastric cancer & $118(40.7)$ & $130(28.6)$ & $<0.001$ & 1.748 & $1.274-2.400$ & 0.001 \\
\hline
\end{tabular}

Values are presented as mean \pm standard deviation or number (\%).

$\mathrm{OR}$, odds ratio; $\mathrm{Cl}$, confidence interval.

Table 4. Risk Factors of Advanced Colorectal Neoplasm of All Study Subjects

\begin{tabular}{|c|c|c|c|c|c|c|}
\hline \multirow{2}{*}{ Characteristics } & \multirow{2}{*}{$\begin{array}{l}\text { Advanced colorectal } \\
\text { neoplasm }(n=96)\end{array}$} & \multirow{2}{*}{$\begin{array}{l}\text { Absent } \\
(n=648)\end{array}$} & \multirow{2}{*}{$P$-value } & \multicolumn{3}{|c|}{ Multivariate analysis } \\
\hline & & & & OR & $95 \% \mathrm{Cl}$ & $P$-value \\
\hline Age (yr) & $63.1 \pm 8.5$ & $62.3 \pm 8.5$ & 0.375 & & & \\
\hline Male sex & 79 (82.3) & $365(56.3)$ & $<0.001$ & 3.620 & $2.092-6.265$ & $<0.001$ \\
\hline Body mass index $\left(\mathrm{kg} / \mathrm{m}^{2}\right)$ & $23.1 \pm 3.0$ & $23.5 \pm 3.3$ & 0.316 & & & \\
\hline Diabetes & $25(26.0)$ & $127(19.6)$ & 0.144 & & & \\
\hline Family history of colorectal cancer & $8(8.3)$ & $34(5.2)$ & 0.215 & & & \\
\hline History of colorectal polyp & $32(33.3)$ & $256(39.5)$ & 0.247 & & & \\
\hline Gastric cancer & $42(43.8)$ & $206(31.8)$ & $<0.001$ & 1.705 & $1.094-2.659$ & 0.019 \\
\hline
\end{tabular}

Values are presented as mean \pm standard deviation or number (\%).

$\mathrm{OR}$, odds ratio; $\mathrm{Cl}$, confidence interval.

2.400) were significant risk factors for CRN. A comparison of individuals with and without ACRN is shown in Table 4. Univariate analysis revealed that male sex and a history of gastric cancer were significant risk factors for ACRN. After multivariate analysis, male sex (OR, 3.620; 95\% CI, 2.092-6.265) and a history of surgery for gastric cancer (OR, 1.705; 95\% CI, 1.0942.659) were identified as significant risk factors. There was no significant difference between patients with and without CRC.

\section{DISCUSSION}

To our knowledge, this study is the first to investigate the usefulness of colonoscopy in gastric cancer patients after gastrectomy. This study confirmed that CRN and ACRN were more prevalent in patients with gastric cancer after gastrectomy than in healthy individuals.

Known risk factors for $\mathrm{CRC}$ are diabetes, hyperlipidemia, alcohol use, and a diet rich in animal fat. ${ }^{21,22}$ The incidence of CRC has been reported to be inversely proportional to the consumption of nonsteroidal anti-inflammatory drugs (NSAIDs) and aspirin. ${ }^{22}$ Our group previously investigated the usefulness of screening colonoscopy in gastric cancer patients before surgery, ${ }^{11}$ and showed that CRN, ACRN, and CRC were more prevalent in the gastric cancer group than in healthy controls. Although CRC is the most common synchronous cancer with gastric cancer, the relationship between these 2 cancers has not been determined. Several studies have suggested that Helicobacter pylori, a causative organism of gastric cancer, is also a risk factor for CRC. ${ }^{23,24}$ In the current study, we compared the prevalence of CRN, ACRN, and CRC between postgastrectomy gastric cancer patients and healthy individuals. The prevalence of CRC was numerically higher in the gastric cancer patients than in the control group, but this difference was not significant. The prevalence of CRN and ACRN was significantly higher in the gastric cancer group than in the healthy controls. In the multivariate analysis, gastric cancer 
was an independent risk factor for CRN and ACRN. This indicates that regular scheduled colonoscopy should be performed after gastrectomy.

In the current study we excluded patients who underwent endoscopic resection. In a previous study, the prevalence of CRN at the time of resection did not differ between the patients who underwent surgical resection and endoscopic resection. ${ }^{11}$ Colonoscopy in patients who underwent gastrectomy is technically demanding because of bowel adhesion. In our center, colonoscopy before gastrectomy and follow up colonoscopy after surgery was usually performed by fellows (gastroenterologist and surgeon). However, in the current study, the colonoscopy in both groups was performed by same experience colonoscopists (professor of gastroenterology) who are low risk for missing adenoma or having chance of interval cancer.

Guidelines recommend an initial screening test for CRC in the general population, starting at age 50, using a stool-based test or endoscopy including colonoscopy and sigmoidoscopy. ${ }^{18}$ Colonoscopy is recognized as the best diagnostic method for detecting CRC and has several benefits. First, as well as detecting CRC, colonoscopy can simultaneously treat a precancerous CRN at the time of diagnosis and hence can prevent CRC. ${ }^{25}$ Most CRNs found at colonoscopy are less than 1 $\mathrm{cm}$ in diameter, and are suitable for conventional methods of polypectomy including hot or cold snare polypectomy. ${ }^{26,27} \mathrm{Sec}-$ ond, colonoscopy can detect metastatic cancer after gastrectomy. We identified metastatic cancer in 3 patients in the gastric cancer group.

We also compared colonoscopy quality measures between the 2 groups. The colonoscope insertion time was longer in the patients who underwent gastrectomy. Colonoscope insertion time is an important technical measure of colonoscopy. ${ }^{28}$ Although the technical difficulty of colonoscope insertion was not recorded by the colonoscopists, the longer insertion time in the gastric cancer group indirectly suggests that colonoscope insertion was more technically demanding in postgastrectomy patients than in the healthy population. We investigated colonoscope withdrawal time only in patients who did not undergo any additional procedures such as a biopsy or polypectomy. Colonoscope withdrawal time is usually defined as the mucosal inspection time excluding time for fluid suction or additional procedures. Thus, it was challenging to analyze colonoscope withdrawal time accurately in patients who underwent polypectomy or a biopsy. In previous studies, the mean inspection time for upper endoscopy or colonoscopy was de- fined as the amount of time that did not result in any positive finding that required additional procedures. ${ }^{19,29}$ In the current study, colonoscope withdrawal time was comparable between the 2 groups. The rate of adenoma detection has been reported to be influenced by withdrawal time, which is an important indicator of adequate colonoscopy. ${ }^{27}$

We acknowledge several limitations of the current study. First, because of its retrospective design, several patients had missing data. Colonoscope withdrawal time was measured in only $50 \%$ of the patients. However, colonoscopy for both groups was performed by the same colonoscopists and withdrawal time was similar for both groups. The use of medications such as aspirin or NSAIDs, which are known to have a protective effect against CRC development, was not analyzed. Second, the control subjects were matched from those who underwent colonoscopy at a health-promotion center, who were relatively healthy and had a strong interest in health screening. Indications for colonoscopy and bowel cleansing quality differed between the 2 groups. A previous history of abdominal surgery is a risk factor for suboptimal bowel cleansing. ${ }^{30}$ However, we excluded patients with inadequate bowel cleansing and therefore believe that these parameters did not affect the main outcome of our study. Third, this study was not designed to investigate the rate of $\mathrm{CRN}$ in the gastric cancer group and the control group.

Despite the limitations described above, our study has several strengths. First, using a well-preserved cohort of surgical data, we included consecutive gastric cancer patients who underwent colonoscopic examination during the study period. Second, colonoscopic examination was performed by experienced endoscopists and quality measures for colonoscopy were met. The rate of adenoma detection was more than $25 \%$ and colonoscope withdrawal time was longer than 6 minutes for both groups.

In conclusion, our study demonstrated that CRN and ACRN were more prevalent in postgastrectomy gastric cancer patients than in healthy individuals. A history of surgery for gastric cancer was an independent risk factor for CRN and ACRN. The results of the current study address the importance of regular surveillance colonoscopy after gastrectomy at appropriate intervals. Future clinical trials are needed to validate the results of this study.

\section{ADDITIONAL INFORMATION}

\section{Funding Source}

The authors received no financial support for the research, 
authorship, and/or publication of this article.

\section{Conflict of Interest}

No potential conflict of interest relevant to this article was reported.

\section{Author Contribution}

Conceptualization: Gweon TG. Data curation: Yoon KT. Formal analysis: Yoon KT. Investigation: Yoon KT. Methodology: Gweon TG, Yoon KT, Kim JJ. Project administration: Gweon TG. Software: Yoon KT. Supervision: Kim CH. Validation: Kim JJ. Writing - original draft: Gweon TG, Yoon KT. Writing - review \& editing: Kim JJ. Approval of final manuscript: all authors.

\section{Others}

We gratefully thank Ha Soon Kang and Fr. Youn Hui Kang Thomas Aquinas.

\section{ORCID}

Gweon TG

Yoon KT

$\mathrm{Kim} \mathrm{CH}$

Kim JJ https://orcid.org/0000-0002-0884-7228 https://orcid.org/0000-0001-9219-9624 https://orcid.org/0000-0003-1567-4318 https://orcid.org/0000-0003-1011-8793

\section{REFERENCES}

1. Jun JK, Choi KS, Lee HY, et al. Effectiveness of the Korean National Cancer Screening Program in reducing gastric cancer mortality. Gastroenterology 2017;152:1319-1328.

2. Allemani C, Matsuda T, Di Carlo V, et al. Global surveillance of trends in cancer survival 2000-14 (CONCORD-3): analysis of individual records for 37513025 patients diagnosed with one of 18 cancers from 322 population-based registries in 71 countries. Lancet 2018;391:1023-1075.

3. Ikeda Y, Saku M, Kawanaka H, Nonaka M, Yoshida K. Features of second primary cancer in patients with gastric cancer. Oncology 2003;65:113-117.

4. Lee JH, Bae JS, Ryu KW, et al. Gastric cancer patients at highrisk of having synchronous cancer. World J Gastroenterol 2006; 12:2588-2592.

5. Eom BW, Lee HJ, Yoo MW, et al. Synchronous and metachronous cancers in patients with gastric cancer. J Surg Oncol 2008; 98:106-110.

6. Saito S, Hosoya Y, Togashi K, et al. Prevalence of synchronous colorectal neoplasms detected by colonoscopy in patients with gastric cancer. Surg Today 2008;38:20-25.
7. Leslie A, Carey FA, Pratt NR, Steele RJ. The colorectal adenoma-carcinoma sequence. Br J Surg 2002;89:845-860.

8. Lieberman DA, Rex DK, Winawer SJ, Giardiello FM, Johnson DA, Levin TR. Guidelines for colonoscopy surveillance after screening and polypectomy: a consensus update by the US Multi-Society Task Force on Colorectal Cancer. Gastroenterology 2012;143:844-857.

9. Choi WS, Han DS, Eun CS, et al. Three-year colonoscopy surveillance after polypectomy in Korea: a Korean Association for the Study of Intestinal Diseases (KASID) multicenter prospective study. Intest Res 2018;16:126-133.

10. Gweon TG, Huh CW, Ji JS, Kim CH, Kim JJ, Park SM. Comparison of bowel-cleansing efficacy of split-dose and same-day dose bowel preparation for afternoon colonoscopy in patients with gastrectomy: a prospective randomized study. Surg Endosc 2020;34:4413-4421.

11. Yoo HM, Gweon TG, Seo HS, et al. Role of preoperative colonoscopy in patients with gastric cancer: a case control study of the prevalence of coexisting colorectal neoplasms. Ann Surg Oncol 2013;20:1614-1622.

12. Washington K. 7th Edition of the AJCC cancer staging manual: stomach. Ann Surg Oncol 2010;17:3077-3079.

13. Huh CW, Gweon TG, Seo M, Ji JS, Kim BW, Choi H. Validation of same-day bowel preparation regimen using $4 \mathrm{~L}$ polyethylene glycol: comparison of morning and afternoon colonoscopy. Medicine (Baltimore) 2018;97:e12431

14. Seo M, Gweon TG, Huh CW, Ji JS, Choi H. Comparison of bowel cleansing efficacy, safety, bowel movement kinetics, and patient tolerability of same-day and split-dose bowel preparation using $4 \mathrm{~L}$ of polyethylene glycol: a prospective randomized study. Dis Colon Rectum 2019;62:1518-1527.

15. Lee HS, Byeon JS. Bowel preparation, the first step for a good quality colonoscopy. Intest Res 2014;12:1-2.

16. Aronchick CA, Lipshutz WH, Wright SH, Dufrayne F, Bergman G. A novel tableted purgative for colonoscopic preparation: efficacy and safety comparisons with Colyte and Fleet Phospho-Soda. Gastrointest Endosc 2000;52:346-352.

17. Gweon TG, Kim SW, Noh YS, et al. Prospective, randomized comparison of same-day dose of 2 different bowel cleanser for afternoon colonoscopy: picosulfate, magnesium oxide, and citric acid versus polyethylene glycol. Medicine (Baltimore) 2015;94:e628.

18. European Colorectal Cancer Screening Guidelines Working Group, von Karsa L, Patnick J, et al. European guidelines for quality assurance in colorectal cancer screening and diagnosis: overview and introduction to the full supplement publica- 
tion. Endoscopy 2013;45:51-59.

19. Barclay RL, Vicari JJ, Doughty AS, Johanson JF, Greenlaw RL. Colonoscopic withdrawal times and adenoma detection during screening colonoscopy. N Engl J Med 2006;355:2533-2541.

20. Kim SY, Kim TI. Serrated neoplasia pathway as an alternative route of colorectal cancer carcinogenesis. Intest Res 2018;16: 358-365.

21. Ferrari P, Jenab M, Norat T, et al. Lifetime and baseline alcohol intake and risk of colon and rectal cancers in the European prospective investigation into cancer and nutrition (EPIC). Int J Cancer 2007;121:2065-2072.

22. Chan AT, Giovannucci EL. Primary prevention of colorectal cancer. Gastroenterology 2010;138:2029-2043.

23. Lee YC, Chiang TH, Chou CK, et al. Association between Helicobacter pylori eradication and gastric cancer incidence: a systematic review and meta-analysis. Gastroenterology 2016; 150:1113-1124.

24. Zumkeller N, Brenner H, Zwahlen M, Rothenbacher D. Helicobacter pylori infection and colorectal cancer risk: a metaanalysis. Helicobacter 2006;11:75-80.

25. Quintero E, Castells A, Bujanda L, et al. Colonoscopy versus fecal immunochemical testing in colorectal-cancer screening. N Engl J Med 2012;366:697-706.

26. Papastergiou V, Paraskeva KD, Fragaki M, et al. Cold versus hot endoscopic mucosal resection for nonpedunculated colorectal polyps sized 6-10 mm: a randomized trial. Endoscopy 2018;50:403-411.

27. Regula J, Rupinski M, Kraszewska E, et al. Colonoscopy in colorectal-cancer screening for detection of advanced neoplasia. $\mathrm{N}$ Engl J Med 2006;355:1863-1872.

28. Jaruvongvanich V, Sempokuya T, Laoveeravat P, Ungprasert P. Risk factors associated with longer cecal intubation time: a systematic review and meta-analysis. Int J Colorectal Dis 2018; 33:359-365.

29. Park JM, Huo SM, Lee HH, Lee BI, Song HJ, Choi MG. Longer observation time increases proportion of neoplasms detected by esophagogastroduodenoscopy. Gastroenterology 2017; 153:460-469.

30. Woo DH, Kim KO, Jeong DE, et al. Prospective analysis of factors associated with inadequate bowel preparation for colonoscopy in actual clinical practice. Intest Res 2018;16:293-298. 